\title{
Effect of Uncertainty on the Reaction Response in Fencing
}

\author{
Marcos Gutiérrez-Dávila and F. Javier Rojas \\ University of Granada \\ Raquel Antonio and Enrique Navarro \\ Polytechnic University of Madrid
}

\begin{abstract}
Purpose: The objective was to determine the way in which the level of uncertainty produced during the execution of a lunge attack with target change (two or four possible responses) affects reaction-response time parameters and kinematic factors involved in the technical coordination of the attack. Method: Seventeen fencers from the Spanish National Men's Fencing Team participated in the study, which involved performing a lunge attack against a moving circle as fast as possible, while attempting to place the tip of the sword inside the circle that appeared in the plastron's center of symmetry. Two force platforms were used to record the anteroposterior component of force and the initiation of movement. Kinematics were measured with a three-dimensional motion-capture system that recorded the spatial positions of 11 markers. An electronic signal was used to time-synchronize all of the systems. Results: The results demonstrate a significant increase in reaction time, $p<.01$, and choice reaction time, $p<.001$, when uncertainty increases, while movement time, precision, and segmental participation sequence during movement are not affected when uncertainty increases. Conclusion: It is worth stressing the importance of uncertainty-reduction strategies for fencers' performance to reduce the reaction-response time. Although increased uncertainty has little influence over precision and segmental participation sequence of the movement pattern used in attacks executed by elite fencers, caution should be used when generalizing these results for fencers of a lower technical level than that of the participants in this study.
\end{abstract}

Keywords: biomechanics, motor control, reaction time, target change

Prior to the execution of attack actions in fencing, a fencer generally performs preparatory movements to predict the opponent's action, reduce his or her response options, or select the appropriate moment for implementing a certain technical action. These actions ultimately aim to reduce the uncertainty of the opponent's potential actions. In addition to facilitating the fencer's attack in anticipation of the opponent's action, reducing uncertainty enables faster information processing, which in turn leads to a faster and more efficient response to the defensive action (Borysiuk \& Waskiewicz, 2008;

Submitted June 19, 2011; accepted March 3, 2012.

Correspondence should be addressed to F. Javier Rojas, Department of Physical Education and Sport, University of Granada, Carretera de Alfacar s/n, 18071 Granada, Spain. E-mail: fjrojas@ugr.es
Czajkowski, 2009). Simple reaction time is widely recognized as being less than choice reaction time (CRT), which increases logarithmically in correlation with the number of the opponent's potential responses (Sanderson, 1983; Schmidt $\&$ Lee, 2011; Stein, 2008). An attacker probes the opponent to reduce the number of possible reactions through preliminary movements. In turn, the attacker has reduced his or her own number of potential responses, resulting in a reduced CRT. However, there is some dispute regarding the comparison of CRT for different levels of uncertainty in fencing. Williams and Walmsley (2000b) did not find differences in CRT for fencers when uncertainty was increased from two to four response options, while Delignières, Brisswalter, and Legros (1994) observed an increase in CRT when the uncertainty was higher. Di Russo, Taddei, Apnile, and Spinelli (2006), 
comparing expert fencers with students, emphasized the expert fencers' ability to quickly determine the correct stimulus and change their movement during the action.

Aside from these arguments, there are few studies in which CRT was recorded during an attack, even though this component is the most common condition in fencing. After provoking target changes among fencers by presenting stimuli up to $200 \mathrm{~ms}$ after the initiation of a movement, Williams and Walmsley (2000a) concluded that the target change did not jeopardize the required technical execution, even in novice fencers. This conclusion is supported by the use of a push-movement pattern based on a kinetic chain model (Kreighbaum \& Barthels, 1996), which enables a fencer to modify the sword's trajectory during the movement's execution. Despite these findings, the technical execution of complex fencing movements is considered to be a highly important factor of fencers' performance (Roi \& Biancheli, 2008; Yiou \& Do, 2000).

Whereas executing a target change is not very difficult for fencers, movement time (MT) must efficiently adapt to the adversary's action and to the time needed to process information while the fencer accelerates forward. Fencers customarily execute lunge attacks at an increasing velocity to reach the target before the front foot lands (Roi \& Biancheli, 2008); therefore, any unforeseen change in CRT produced by the opponent's movements could alter the execution's timing of the attacker, and consequently, the segmental participation sequence. In this way, a change in CRT as a result of increased uncertainty would involve certain modifications during the movement's execution, and the difficulty of coordinating the technical action would increase.

The objective of this study was to determine the effect of uncertainty on reaction-response time (RRT) parameters by recording CRT during the execution of a lunge attack with a target change. To this end, two levels of uncertainty were set up, in accordance with the number of possible responses (2CRT and 4-CRT, for two and four responses, respectively). Despite the disputes surrounding this issue, we hypothesized the existence of statistically significant differences in the time used for information processing, which will be greatest in the condition of increased uncertainty (4-CRT). If, as we expect, CRT increases with increased uncertainty, then we also expect that certain kinematic variables related to the segmental participation sequence will be affected.

\section{METHOD}

Seventeen expert fencers from the Spanish National Men's Fencing Team, with more than 9 years of experience (mean values: age $=23.3 \pm 4.1$ years old; height $=1.83 \pm 0.05 \mathrm{~m}$, weight $=78 \pm 8 \mathrm{~kg}$ ), participated in this study. Informed consent was obtained from all participants, in keeping with the guidelines established by the University of Granada Ethics Committee, which approved the authors' study.
For the lunge attack, the fencers used their personal swords equipped with a digital 1/1,000-s stopwatch interfaced with their wiring system. The chronometers recorded the times when the tip of the sword made contact with a $0.7 \mathrm{~m} \times 0.55 \mathrm{~m}$ white screen acting as a plastron (target). The fencers adopted their usual en-garde position, placing the big toe of their back foot at a distance 1.5 times greater than their height, with respect to the plastron. After executing several attack actions against the plastron from this pre-established point, the fencers were allowed to make adjustments as necessary until finding a comfortable distance. The fencers then increased the distance to the plastron by an average of $0.06 \pm 0.22 \mathrm{~m}$, and this new distance was maintained for all trials.

A projector linked to a computer with an external programmable card was used to control the timing of the projection of a black circle with a $0.09-\mathrm{m}$ diameter on the plastron (see Figure 1). The system was designed so that the circle would appear after a random period of time ranging from $0.5 \mathrm{~s}$ to $1.2 \mathrm{~s}$ after the start command. Following this period, an electronic signal synchronized the circle's projection with the initiation of the chronometer, which

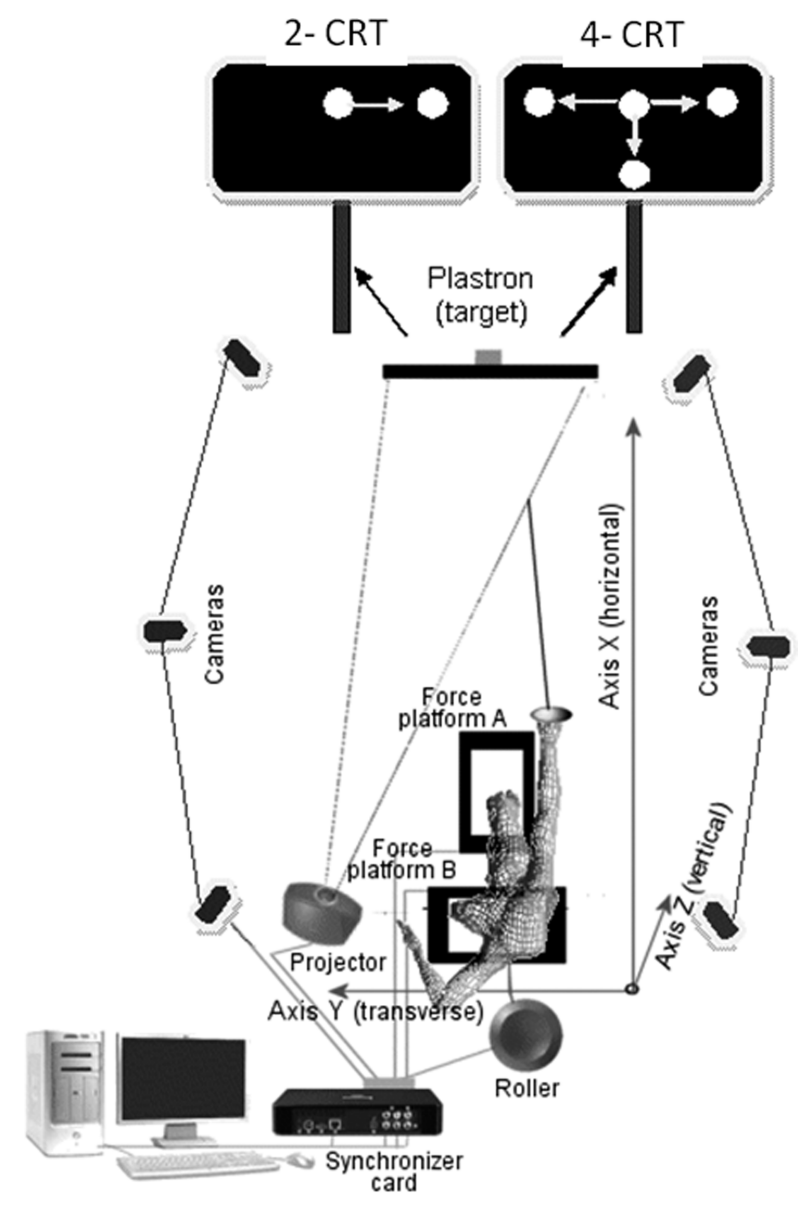

FIGURE 1 Illustration of the recording systems used for the two experimental conditions, 2-CRT and 4-CRT. 
was then stopped by the contact of a sensor attached to the tip of the sword with the circle. The fencers were told to remain still in the en-garde position until the projected circle appeared on the plastron. From this moment on, the fencers were instructed to execute a direct attack as fast as possible, while positioning the tip of the sword inside the circle. Before beginning the recorded trials, the participants executed a session of several attacks to become familiarized with the system.

For the trials, eight body markers were situated on each fencer-on the two heels and the front tips of both feet, and the knee (epicondylus fibularis femoris), the hip (trochanter major), the shoulder (tuberculum majus), and the elbow (epicondylus radialis) of the sword arm-and three markers situated on the blade of the sword (first third, second third, and point).

A three-dimensional analysis system with six VICON460 infrared video cameras at $500 \mathrm{~Hz}$ was used to record the spatial positions of the 11 markers. The axes that define the positions are shown in Figure 1 . Two $0.6 \mathrm{~m} \times 0.37 \mathrm{~m}$ Dinascan/IBV force platforms (Biomechanics Institute of Valencia, Spain), at $500 \mathrm{~Hz}$, were placed under the fencers' feet in the en-garde position to record the horizontal component of the reaction force $\left(\mathrm{F}_{\mathrm{AX}}\right.$ and $\left.\mathrm{F}_{\mathrm{BX}}\right)$, parallel to the direction of the lunge. Following the method of Gutiérrez-Dávila, Dapena, and Campos (2006), the start of the movement was determined from the instant at which the net force of the horizontal component $\left(\mathrm{F}_{\mathrm{AX}}+\mathrm{F}_{\mathrm{BX}}\right)$ reached a value greater than or equal to $1 \%$ of the participant's body weight. When this time was less than $100 \mathrm{~ms}$, the trial was repeated because it suggested anticipation. To eliminate any possible systematic errors, the baseline was determined by measuring the mean of the first 100 samples we received from the platform, where the fencer was motionless, ready to begin the movement. The same electronic signal that initiated the chronometer linked to the sword was used to time-synchronize all of the abovementioned recording systems, as illustrated in Figure 1.

\section{Simple Reaction Time}

In the first phase of data acquisition, the fencers were instructed to respond to a circle's appearance in the plastron's center of symmetry with a direct lunge attack. After several practice trials to become acclimated to the condition, the fencers performed five valid trials, in which RRT was recorded and errors (failure of the tip of the sword to impact the circle) were noted. Reaction time (RT) was defined as the period between the circle's appearance in the center of the plastron $\left(S_{1}\right)$ and the initiation of movement $\left(t_{\mathrm{S} 1}\right)$ determined by the moment in which the net horizontal force $\left(\mathrm{F}_{\mathrm{AX}}+\mathrm{F}_{\mathrm{BX}}\right)$ reached a value greater than or equal to $1 \%$ of the participant's body weight. We stipulated that a false start occurs when the RT is less than $100 \mathrm{~ms}$ of a second; in this case, the trial was repeated. This rule is the same as that of the International Association of Athletics Federation for a false start in a sprinter of $100 \mathrm{~m}$ and is the same as that used by Gutiérrez-Dávila et al. (2006). When this period was shorter than $100 \mathrm{~ms}$, the trial was repeated. The chronometer linked to the sword recorded MT, which was obtained based on RRT (MT = RRT $-\mathrm{RT})$.

\section{Choice Reaction Time}

After recording the five valid trials from the first phase, trials were performed in the two experimental conditions (2CRT and 4-CRT). As in the previous condition, the fencers began from the en-garde position and executed a lunge as fast as possible, while attempting to place the tip of the sword inside the circle that appeared in the plastron's center of symmetry. The 2-CRT condition involved the possibility of a shift in the circle's position to a point established $0.25 \mathrm{~m}$ to the right of the initial position before the end of the reaction response. In the 4-CRT condition, the circle could randomly shift toward three equidistant positions located on both sides of and below the initial position, at a distance of $0.25 \mathrm{~m}$ (see Figure 1). The moment at which the circle changed position $\left(t_{\mathrm{S} 2}\right)$, second stimulus $\left(\mathrm{S}_{2}\right)$, was determined based on the median value of RRT from the five valid trials performed in the first phase, where $t_{\mathrm{S} 2}=\mathrm{RT}+$ (MT/4). The execution order for the two conditions alternated among the fencers.

In the five valid trials for each of the experimental conditions, a stimulus change was introduced at the $t_{\mathrm{S} 2}$ moment. To minimize the learning effect, the fencers also participated in two trials where no change was produced and two trials in which the stimulus change occurred at a random time between $t_{\mathrm{S} 1}$ and $t_{\mathrm{S} 2}$. The order of the trials was random, and those that did not reach the target were repeated and marked as errors. Only the five valid trials where the target change was produced in $t_{\mathrm{S} 2}$ were recorded, and the median value of RRT was analyzed in each experimental condition, 2-CRT and 4-CRT. Before beginning the data acquisition process, the fencers completed several trials to become familiarized with this new testing condition.

Because the target change was produced during MT, this unit was divided into three epochs. The first was designated "MT-stimulus change" and was defined as the period between the initiation of movement $\left(t_{\mathrm{S} 1}\right)$ and the moment of the stimulus change $\left(t_{\mathrm{S} 2}\right)$. CRT was then recorded and consisted of the period of time between $t_{\mathrm{S} 2}$ and the moment at which the sword's trajectory changed toward the new objective $\left(t_{\mathrm{TC}}\right)$. The change produced in the transverse or vertical component of acceleration of the marker on the end of the sword's forte (depending on whether the sword's displacement was lateral or downward, respectively) was used to determine $t_{\mathrm{TC}}$. Figure 2 shows a typical example of the kinematics of the sword with a depiction of the trajectory change. For the calculation of the acceleration 
components, the second derivative of the movement vector was used through fifth-grade splines without applying the smoothing function. Although the marker on the tip of the sword was more sensitive to change, it was rejected due to the possibility of the creation of interferences by movements resulting from the sword's elasticity. Finally, "MT-target change" was defined as the period of time between $t_{\mathrm{TC}}$ and the execution of the touché.

The horizontal acceleration of the center of mass (CM) was calculated from the net horizontal force of the two platforms $\left(\mathrm{F}_{\mathrm{AX}}\right.$ and $\left.\mathrm{F}_{\mathrm{BX}}\right)$ and the fencer's mass. The horizontal velocities $\left(\boldsymbol{v}_{\mathrm{x}(\mathrm{CM})}\right)$ and displacements $\left(\boldsymbol{s}_{\mathrm{X}(\mathrm{CM})}\right)$ were calculated from the horizontal acceleration-time values using trapezoidal integration. To calculate the horizontal velocity and displacement components of the markers, the first derivative was applied to the corresponding components of the position with respect to time. The angles of the flexion of the elbow $\left(\boldsymbol{\theta}_{\text {(ELBOw) }}\right)$ and the shoulder $\left(\boldsymbol{\theta}_{\text {(SHOULDER })}\right)$ were calculated using the scalar product of the vectors that determined the positions of the markers defining the respective segments, (shoulder-hip/ shoulder-elbow, for the shoulder; and elbow-shoulder/ elbow beginning of the forte of the blade, for the elbow angle). The length of the lunge was defined as the distance between the horizontal coordinate of the marker on the back foot in the en-garde position and the marker on the heel of the front foot when fully rested after executing the lunge.
Data are expressed as means $(M)$ and standard deviations (SD) for each experimental variable and condition. A repeated-measures analysis of variance (ANOVA) was used to verify statistically significant differences between the two conditions. The level for acceptance of significance $(\alpha)$ was set at .05. Mean differences between conditions and 95\% confidence intervals (CI) were calculated. Effect-size statistics were assessed using Cohen's d (Nakagawa \& Cuthill, 2007). Taking into account the cutoff established by Cohen, the effect size can be small $(\approx .2)$, medium $(\approx .5)$, or large $(\approx .8)$. To evaluate the reliability of the trials, an ANOVA for repeated measures was applied to all the trials under the two experimental conditions (five trials in each condition), taking as the dependent variables the RRT, MT, and RT. The intraclass correlation coefficients were .946 and .963 for RRT, .745 and .837 for RT, and .968 and .933 for TM for the two experimental conditions, 2-CRT and 4-CRT, respectively (all $p<.001$ ). Results were analyzed using the Statistical Package for the Social Sciences, SPSS v. 15.0 software for Windows.

\section{RESULTS}

Figure 3 shows the individual values of horizontal force with respect to time in the two experimental conditions (2-CRT and 4-CRT). This figure demonstrates that the

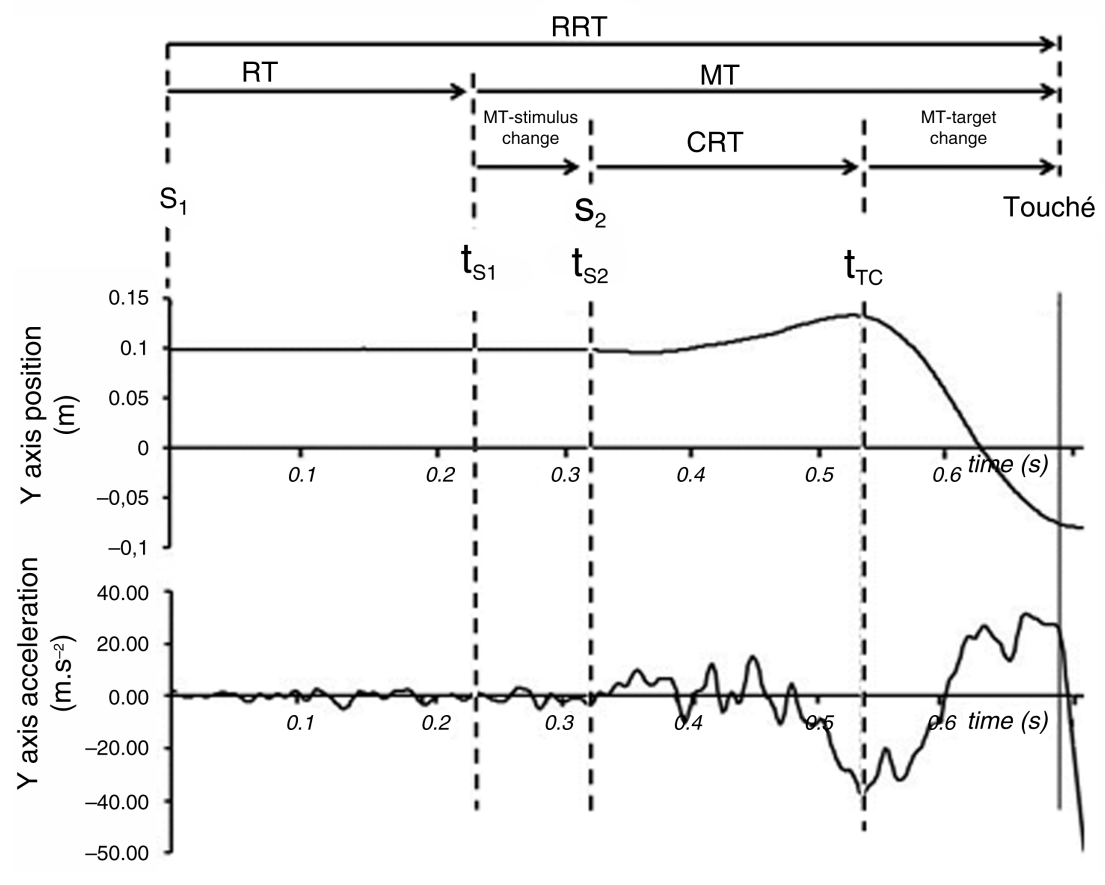

FIGURE 2 Typical kinematics of the sword with a depiction of the trajectory change. The reaction-response time components are shown above the graph. $\mathrm{CRT}=$ choice reaction time; $\mathrm{S}_{1}=$ initial stimulus; $\mathrm{S}_{2}=$ second stimulus; $\mathrm{MT}=$ movement time; MT-stimulus change $=$ movement time-stimulus change; MT-target change $=$ movement time-target change; RRT $=$ reaction-response time; RT $=$ reaction time; $t_{\mathrm{S} 1}=$ instant of beginning of the movement; $t_{\mathrm{S} 2}=$ instant of the stimulus change; $t_{\mathrm{TC}}=$ instant when the fencer changes the direction of the sword toward the new target. 
horizontal acceleration impulse (acceleration phase) is greater for the 2-CRT condition than for the 4-CRT condition. Initiation of movement $\left(t_{\mathrm{S} 1}\right)$ occurs later in the 4CRT condition although acting similarly in both conditions. After the target change $\left(t_{\mathrm{S} 2}\right)$, the horizontal force increases until reaching its maximum peak, which is very close to the moment of the change $\left(\mathrm{t}_{\mathrm{TC}}\right)$. In both of the experimental conditions, the fencer increases the horizontal force while processing information (CRT).

The CRT that is produced during the application of forces is longer for the 4-CRT condition, causing the target change to be initiated after the horizontal force reaches its maximum peak. The figure also shows that the touché is produced after the acceleration phase, at the end of the flight phase. Although the figure corresponds to one of the analyzed participants, this description may be considered as general behavior with the analysis of the numerical data presented.

Table 1 shows the RRT parameters for the 2-CRT and 4CRT conditions. RRT was significantly shorter for the 2CRT condition. Although the initial stimulus was identical and was presented in the same initial condition, our findings revealed that RT was significantly longer for the 4-CRT condition (199 ms vs. $223 \mathrm{~ms}$ ). However, no statistically significant differences were found for MT in the two experimental conditions.

The average time between the initiation of movement, $t_{\mathrm{S} 1}$, and the execution of the target change, $t_{\mathrm{S} 2}, M T$-stimulus change, was significantly shorter for the 4-CRT condition (180 ms vs. $150 \mathrm{~ms}$ ) because the RT was significantly longer for the 4-CRT condition and the MT was similar for both experimental conditions. CRT was significantly longer for the 4-CRT condition (186 ms vs. $243 \mathrm{~ms}$ ), while there were no statistically significant differences for the MT composed between the initiation of the sword's change in trajectory, $t_{\mathrm{TC}}$, and the moment when the sword came into contact with the plastron, touché.

Errors were expressed in percentages of all the trials performed in the 2-CRT and 4-CRT conditions. In contrast with our expectations, no differences were found in the two experimental conditions. At the end of the acceleration phase, the horizontal velocity and displacement of the CM $\left(\boldsymbol{v}_{\mathrm{x}(\mathrm{CM})}\right.$ and $\boldsymbol{s}_{\mathrm{x}(\mathrm{CM})}$, respectively) were significantly greater for the 2-CRT condition $\left(1.97 \mathrm{~ms}^{-1}\right.$ vs. $1.80 \mathrm{~ms}^{-1}$ and $0.41 \mathrm{~m}$ vs. $0.37 \mathrm{~m}$, respectively). At the moment of the stimulus change, the horizontal velocity and displacement of the CM $\left(\boldsymbol{v}_{\mathrm{x}(\mathrm{CM})}\right.$ stimulus change and $\boldsymbol{s}_{\mathrm{x}(\mathrm{CM})}$ stimulus change, respectively) were significantly greater for the 2CRT condition $\left(0.35 \mathrm{~ms}^{-1}\right.$ vs. $0.26 \mathrm{~ms}^{-1}$ and $0.03 \mathrm{~m}$ vs. $0.02 \mathrm{~m}$, respectively). No statistically significant differences were found for these two variables at the moment when the target change was produced $\left(t_{\mathrm{TC}}\right)$.

Table 2 contains the kinematic variables for the initial, en-garde position in the two experimental conditions. The central-tendency data are presented to describe the characteristics of the sequence of segmental participation during MT. The time records are expressed as a percentage of the acceleration phase. No statistically significant differences were found between the two experimental conditions for any of the variables describing the en-garde position; therefore, the average initial position was similar for both conditions (2-CRT and 4-CRT). For variables of

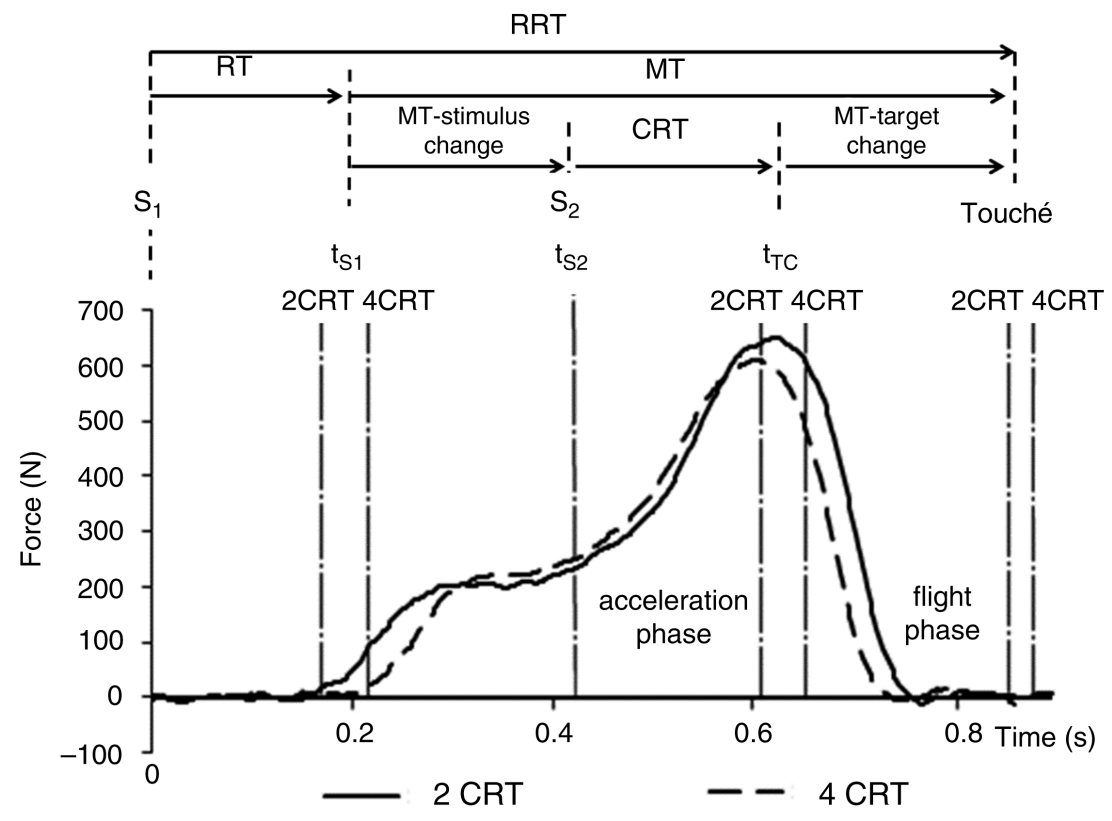

FIGURE 3 Graphical representation of the force with respect to time for the two experimental conditions (2-CRT and 4-CRT) for one of the fencers analyzed. 
TABLE 1

Descriptive and Inferential Statistics for Reaction-Response Time Parameters and Other Relevant Variables for the Two Experimental Conditions (2-CRT and 4-CRT)

\begin{tabular}{|c|c|c|c|c|c|}
\hline Variables & $2-C R T$ Mean $\pm S D$ & 4-CRT Mean $\pm S D$ & Mean Differences & $95 \% C I$ & Effect Size $(d)$ \\
\hline Response-Reaction Time, RRT (ms) & $793 \pm 85 * *$ & $826 \pm 95 * *$ & $-35 \pm 46$ & -59 to -12 & 0.8 \\
\hline Reaction Time, RT (ms) & $199 \pm 22 * *$ & $223 \pm 31 * *$ & $-24 \pm 27$ & -38 to -10 & 0.9 \\
\hline Movement Time, MT (ms) & $592 \pm 83$ & $604 \pm 88$ & $-13 \pm 46$ & -36 to 11 & 0.3 \\
\hline $\mathrm{MT}-$ stimulus change, $\mathrm{MT}-\mathrm{T}_{\mathrm{S} 2}(\mathrm{~ms})$ & $180 \pm 57 * *$ & $150 \pm 49 * *$ & $31 \pm 35$ & 13 to 49 & 0.9 \\
\hline Choice Reaction Time, CRT (ms) & $186 \pm 46 * * *$ & $243 \pm 44 * * *$ & $-57 \pm 33$ & -74 to -40 & 1.8 \\
\hline MT target change, $\mathrm{MT}-\mathrm{T}_{\mathrm{TC}}(\mathrm{ms})$ & $224 \pm 56$ & $211 \pm 62$ & $14 \pm 50$ & -12 to 40 & 0.3 \\
\hline Acceleration phase (ms) & $530 \pm 82$ & $536 \pm 97$ & $-6 \pm 56$ & -34 to 23 & 0.1 \\
\hline Flight phase (ms) & $35 \pm 33$ & $23 \pm 47$ & $12 \pm 39$ & -8 to 33 & 0.3 \\
\hline Errors $(\%)$ & $30 \pm 17$ & $34 \pm 12$ & $-4 \pm 14$ & -11 to 3 & 0.3 \\
\hline $\boldsymbol{v}_{\mathrm{X}(\mathrm{CM})}$ at end of acceleration $\left(\mathrm{m} \cdot \mathrm{s}^{-1}\right)$ & $1.97 \pm 0.32 * *$ & $1.80 \pm 0.40 * *$ & $0.17 \pm 0.15$ & 0.09 to 0.2 & 1.1 \\
\hline$s_{\mathrm{X}(\mathrm{CM})}$ at end of acceleration $(\mathrm{m})$ & $0.41 \pm 0.08 *$ & $0.37 \pm 0.09 *$ & $0.04 \pm 0.06$ & 0.01 to 0.07 & 0.6 \\
\hline $\boldsymbol{v}_{\mathrm{X}(\mathrm{CM})}$ at stimulus change, $t_{\mathrm{S} 2}\left(\mathrm{~m} \cdot \mathrm{s}^{-1}\right)$ & $0.35 \pm 0.20 * *$ & $0.26 \pm 0.21 * *$ & $0.09 \pm 0.1$ & 0.03 to 0.14 & 0.8 \\
\hline$s_{\mathrm{X}(\mathrm{CM})}$ at stimulus change, $t_{\mathrm{S} 2}(\mathrm{~m})$ & $0.03 \pm 0.02 * *$ & $0.02 \pm 0.02 * *$ & $0.01 \pm 0.01$ & 0.00 to 0.01 & 0.9 \\
\hline $\boldsymbol{v}_{\mathrm{X}(\mathrm{CM})}$ at target change, $\mathrm{t}_{\mathrm{TC}}\left(\mathrm{m} \cdot \mathrm{s}^{-1}\right)$ & $1.23 \pm 0.54$ & $1.15 \pm 0.53$ & $0.08 \pm 0.3$ & -0.06 to 0.21 & 0.3 \\
\hline$s_{\mathrm{X}(\mathrm{CM})}$ at target change, $\mathrm{t}_{\mathrm{TC}}(\mathrm{m})$ & $0.17 \pm 0.10$ & $0.16 \pm 0.09$ & $0.01 \pm 0.05$ & -0.02 to 0.03 & 0.1 \\
\hline
\end{tabular}

$* p<.05 . * * p<.01 . * * * p<.001$.

the segmental participation sequence, statistically significant differences were found only for the maximum velocity reached by the sword during MT. This velocity was greater for the 2-CRT condition $\left(2.56 \mathrm{~ms}^{-1}\right.$ vs. $\left.2.37 \mathrm{~ms}^{-1}\right)$, which may be related to the greater $\mathrm{CM}$ velocity obtained in the
2-CRT position at the end of the acceleration phase (see Table 1). It seems that the two experimental conditions did not affect the segmental participation sequence of the movement pattern used in the lunge attack with target change.

TABLE 2

Descriptive and Inferential Statistics for the Most Relevant Kinematic Variables for the Experimental Conditions (2-CRT and 4-CRT)

\begin{tabular}{|c|c|c|c|c|c|}
\hline Variables & 2-CRT Mean $\pm S D$ & 4-CRT Mean $\pm S D$ & Mean Differences & $95 \% C I$ & Effect Size $(d)$ \\
\hline \multicolumn{6}{|l|}{ 'En-Garde' Position } \\
\hline Hip horizontal position (m) & $0.39 \pm 0.06$ & $0.38 \pm 0.05$ & $0.01 \pm 0.04$ & -0.01 to 0.03 & 0.2 \\
\hline Hand horizontal position (m) & $1.08 \pm 0.09$ & $1.08 \pm 0.09$ & $0.00 \pm 0.06$ & -0.03 to 0.03 & 0.0 \\
\hline $\boldsymbol{\theta}_{\text {(ELBOW) }}\left({ }^{\circ}\right)$ & $102 \pm 13$ & $109 \pm 14$ & $-6 \pm 14$ & -13 to 1 & 0.4 \\
\hline $\boldsymbol{\theta}_{\text {(SHOULDER) }}\left({ }^{\circ}\right)$ & $45 \pm 8$ & $45 \pm 11$ & $-1 \pm 7$ & -5 to 2 & 0.2 \\
\hline $\boldsymbol{v}_{\mathrm{X}(\mathrm{MAX})}$ arm extension $\left(\mathrm{m} . \mathrm{s}^{-1}\right)$ & $1.90 \pm 0.51$ & $1.91 \pm 0.59$ & $0.00 \pm 0.4$ & -0.19 to 0.19 & 0.0 \\
\hline$\theta_{\text {(ELBOw) }}$ at maximum velocity of arm $\left(^{\circ}\right)$ & $118 \pm 8$ & $124 \pm 10$ & $-4 \pm 14$ & -11 to 3 & 0.3 \\
\hline $\boldsymbol{\theta}_{(\text {SHOULDER) }}$ maximum velocity of arm $\left(^{\circ}\right)$ & $62 \pm 7$ & $65 \pm 10$ & $-3 \pm 7$ & -6 to 1 & 0.4 \\
\hline Time $v_{\mathrm{X}(\mathrm{MAX})}$ foot $(\%)$ & $70 \pm 9$ & $70 \pm 10$ & $0 \pm 7$ & -4 to 4 & 0.0 \\
\hline $\boldsymbol{v}_{\mathrm{X}(\mathrm{MAX})}$ foot $\left(\mathrm{m} \cdot \mathrm{s}^{-1}\right)$ & $4.59 \pm 0.61$ & $4.38 \pm 0.77$ & $0.20 \pm 0.4$ & -0.02 to 0.42 & 0.5 \\
\hline $\boldsymbol{\theta}_{\text {(ELBOW) }}$ at maximum velocity of foot $\left(^{\circ}\right)$ & $159 \pm 15$ & $159 \pm 17$ & $1 \pm 7$ & -3 to 5 & 0.1 \\
\hline $\boldsymbol{\theta}_{(\text {SHOULDER) }}$ maximum velocity sword $\left(^{\circ}\right)$ & $103 \pm 11$ & $105 \pm 10$ & $-2 \pm 5$ & -4 to 1 & 0.3 \\
\hline $\boldsymbol{v}_{\mathrm{X}}$ sword at touché $\left(\mathrm{m} \cdot \mathrm{s}^{-1}\right)$ & $2.15 \pm 0.65$ & $2.28 \pm 0.47$ & $-0.12 \pm 0.41$ & -0.33 to 0.09 & 0.3 \\
\hline Lunge length $(\mathrm{m})$ & $1.39 \pm 0.17$ & $1.34 \pm 0.18$ & $0.04 \pm 0.1$ & -0.01 to 0.09 & 0.4 \\
\hline
\end{tabular}

Note. Time $v_{\mathrm{x}(\mathrm{MAX})}$ arm extension $(\%)=$ beginning of arm extension; $\boldsymbol{v}_{\mathrm{X}}$ arm extension $\left(\mathrm{m} . \mathrm{s}^{-1}\right)=$ sword horizontal velocity at beginning of arm extension; Time $v_{\mathrm{X}(\mathrm{MAX})}$ foot $(\%)=$ time for the advanced foot to reach the maximum velocity; $\mathrm{V}_{\mathrm{x}(\mathrm{MAX})}$ foot $\left(\mathrm{m} . \mathrm{s}^{-1}\right)=$ foot maximum horizontal

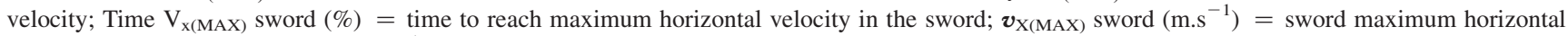
velocity; and $\boldsymbol{v}_{\mathrm{X}}$ sword at touché $\left(\mathrm{m} . \mathrm{s}^{-1}\right)=$ sword horizontal velocity at touché.

$* p<.05$. 


\section{DISCUSSION}

The study's results reveal that the average RRT was significantly longer for the 4-CRT condition. As no statistically significant differences were found for MT, the differences recorded for RRT must have been a result of RT. Indeed, the data demonstrate that the average RT was significantly longer for the 4-CRT condition, a result that we did not expect, as the fencers responded to the same initial stimulus for the two experimental conditions (the appearance of a circle in the plastron's center of symmetry). Given the conditions, the differences recorded between the RT averages must be due to the subsequent uncertainty. Therefore, RT not only increases in correlation with the number of possible responses, as stated by Hick's law for CRT (Hick, 1952), but it also increases when the fencer predicts that a stimulus change may occur during MT, as demonstrated in the two experimental conditions in this study. The RT data gathered in the two experimental conditions and the greater CRT in the 4-CRT condition supports this conclusion and suggests that RT would be influenced by uncertainty as a result of the number of possible responses to the subsequent target change.

Although CRT was recorded during movement in this study, the results confirm general theories maintaining that CRT increases in correlation with the number of responses (Schmidt \& Lee, 2011). In fact, the average data recorded for CRT have significantly greater values for the 4-CRT condition than the 2-CRT condition $(243 \mathrm{~ms}$ vs. $186 \mathrm{~ms}$, respectively, $p<.001)$. Our findings also coincide with the general results obtained by Delignières et al. (1994) through increasing a fencer's uncertainty from two to four response options. MT was divided into three periods of time, including CRT (MT-stimulus change, CRT, and MT-target change). The partial records of these three periods explain why there were no statistically significant differences in MT. MTstimulus change was significantly longer for the 2-CRT condition, while the opposite was true for CRT, and no differences were found between the MT-target change averages.

MT-stimulus change was longer for the 2-CRT condition as a consequence of the differences between the RT averages. As the moment of the stimulus change $\left(t_{\mathrm{S} 2}\right)$ is the same for both conditions and the average RT is longer for the 4-CRT condition, the average MT-stimulus change value would necessarily be lower for the same experimental condition (see Figure 2). The significantly shorter $M T$ stimulus change for the 4-CRT condition may explain the differences between the averages recorded for the velocity and horizontal displacement of the $\mathrm{CM}$ during this period $\left(\boldsymbol{v}_{\mathrm{X}(\mathrm{CM})}\right.$ and $\boldsymbol{s}_{\mathrm{X}(\mathrm{CM})}$, respectively), which also yielded lower values for the 4-CRT condition. As explained earlier, the average RRT was significantly shorter for the 2-CRT condition due to the two periods of time necessary for processing information (RT and CRT).
The average velocity and horizontal displacement of the $\mathrm{CM}$ at the end of the acceleration phase $\left(\boldsymbol{v}_{\mathrm{X}(\mathrm{CM})}\right.$ and $\boldsymbol{s}_{\mathrm{X}(\mathrm{CM})}$ end of the acceleration phase, respectively), was significantly greater for the 2-CRT condition. As no statistically significant differences were found between the averages of these data during the target change's execution $\left(\boldsymbol{v}_{\mathrm{X}(\mathrm{CM})}\right.$ and $\boldsymbol{s}_{\mathrm{X}(\mathrm{CM})}$ target change, respectively), we can confirm that the average $\mathrm{CM}$ acceleration during the MT-target change period is greater when there are fewer possible responses (2CRT). Figure 3 illustrates this phenomenon with a representation of the horizontal force with respect to time. Although this figure only corresponds to one of the fencers, the numerical data it contains show why the $\mathrm{CM}$ acceleration is greater when uncertainty is reduced. As previously explained, the average time used to process information during the attack's execution (CRT) was significantly shorter for the 2-CRT condition, which advances the moment of the target change's initiation $\left(t_{\mathrm{S} 2}\right)$ in relation to the 4-CRT condition. This fact, along with a greater maximum horizontal force recorded in the 2-CRT condition, causes the horizontal impulse during MT-target change to increase when uncertainty is reduced (see Figure 3). In this sense, Cronin, McNair, and Marshall (2003) emphasize the importance of the maximum peak of force in fencers' performance, which our study confirms by finding that this factor contributes to the increase in acceleration at the end of the movement.

In keeping with our expectations, the data shown in Table 2 suggest a similar initial position for both of the conditions presented. The variables that measure the segmental participation sequence during movement confirm that the sword's maximum horizontal velocity ( $\boldsymbol{v}_{\mathrm{X}(\mathrm{MAX})}$ sword) is greater when uncertainty is reduced, perhaps as a result of the greater horizontal velocity of the $\mathrm{CM}$ at the end of the acceleration phase. Therefore, despite the occurrence of time changes during the attack's execution as a result of the periods of time used for processing information (RT and CRT), there is no evidence that these changes affect the segmental participation sequence. The low level of difficulty involved in the lunge attack's movement pattern supports this conclusion, as does the work of Williams and Walmsley (2000a), confirming the lack of technical difficulty posed by a target change. Regardless of these findings, the sample used in this study was composed of elite fencers with an unusually high technical execution level. These results cannot be generalized for fencers of a lower technical level, especially when certain studies highlight the importance of technical execution for complex fencing movements (Williams \& Walmsley, 2000b; Yiou \& Do, 2000). Thus, this aspect offers a future research opportunity.

This study demonstrates that when the number of possible responses during a lunge attack is increased, RRT increases as a result of the periods of time used to process information (RT and CRT), while precision and segmental 
participation sequence are not affected. Therefore, it is worth stressing the importance of uncertainty-reduction strategies for fencers' performance. Although increased uncertainty has little influence over precision and segmental participation sequence of the movement pattern used in attacks executed by elite fencers, caution should be used when generalizing these results for fencers of a lower technical level than that of the participants in this study.

\section{WHAT DOES THIS ARTICLE ADD?}

The results confirm general theories maintaining that CRT increases in correlation with number of responses (Schmidt \& Lee, 2011). However, the fact that CRT was recorded during the execution of a lunge attack, a complex movement in fencing, allows us to generalize the theory and verify that, for expert fencers, the time used for information processing does not affect the segmental participation sequence.

Despite the fact that the RT to the first stimulus was not the main objective of this study, it was, surprisingly, significantly longer when the number of possible responses for the second stimuli increased. This conclusion suggests that RT is influenced by uncertainty. The explanation that we might make for this finding from the perspective of neuropsychology constitutes an aspect of special relevance for future research. Cognitive neuropsychology recognizes that to guarantee adaptability of answers that imply uncertainty and to increase the precision of the response, higher-order automatic facilitation that disables the first action unleashed by a stimulus is needed (Gao, Wong-Lin, Holmes, Simen, \& Cohen, 2009). This necessity may produce a certain delay in the RT.

With regard to practical application, we assert that prior knowledge of the opponent's possible response allows a reduction in uncertainty and RT as well as a quicker execution of the direct thrust despite target change, which constitutes a tactical element of special relevance in fencing.

\section{ACKNOWLEDGMENTS}

The authors wish to thank Cristina López de Subijana for her invaluable help collecting data and the Spanish Royal Federation of Fencing for its collaboration in this project, providing access to the fencers of the National Epee Fighting Team.

\section{REFERENCES}

Borysiuk, Z., \& Waskiewicz, Z. (2008). Information processes, stimulation and perceptual training in fencing. Journal of Human Kinetics, 19, 63-82.

Cronin, J., McNair, P. J., \& Marshall, R. N. (2003). Lunge performance and its determinants. Journal of Sports Sciences, 21, 49-57.

Czajkowski, Z. (2009). Tactics in fencing: Preparatory actions. Studies in Physical Culture and Tourism, 16, 371-378.

Delignières, D., Brisswalter, J., \& Legros, P. (1994). Influence of physical exercise on choice reaction time in sports experts: The mediating role of resource allocation. Journal of Human Movement Studies, 27, 173-188.

Di Russo, F., Taddei, F., Apnile, T., \& Spinelli, D. (2006). Neural correlates of fast stimulus discrimination and response selection in top-level fencers. Neuroscience Letters, 408, 113-118.

Gao, J., Wong-Lin, K., Holmes, P., Simen, P., \& Cohen, J. D. (2009). Sequential effects in two-choice reaction time tasks: Decomposition and synthesis of mechanisms. Neural Computation, 21, 2407-2436.

Gutiérrez-Dávila, M., Dapena, J., \& Campos, J. (2006). The effect of muscular pre-tensing on the sprint start. Journal of Applied Biomechanics, 22, 194-201.

Hick, W. E. (1952). On the rate of gain of information. Quarterly Journal of Experimental Psychology, 4, 11-26.

Kreighbaum, E., \& Barthels, K. M. (1996). Biomechanics: A qualitative approach for studying human movement (4th ed.). Boston: Allyn \& Bacon.

Nakagawa, S., \& Cuthill, I. C. (2007). Effect size, confidence interval and statistical significance: A practical guide for biologists. Biological Reviews Cambridge Philosophical Society, 82, 591-605.

Roi, G. S., \& Biancheli, D. (2008). The science of fencing: Implications for performance and injury preventions. Sports Medicine, 38, 462-481.

Sanderson, F. H. (1983). The effect of directional uncertainty on reaction time and movement time in a fencing task. Journal of Sports Sciences, 1 , 100-105.

Schmidt, R. A., \& Lee, T. D. (2011). Motor control and learning (5th ed.). Champaign, IL: Human Kinetics.

Statistical Package for the Social Studies (Version 15.0). [Computer software]. Chicago: SPSS, Inc.

Stein, J. F. (2008). Factors influencing the initiation, performance and precision of the hit in fencing. In X. Iglesias (Ed.), Proceedings of the 1st International Congress on Science and Technology in Fencing (pp. 7-13). Barcelona, Spain: Generalitat de Catalunya.

Williams, L. R. T., \& Walmsley, A. (2000a). Response amendment in fencing: Differences between elite and novice subjects. Perceptual and Motor Skills, 91(1), 131-142.

Williams, L. R. T., \& Walmsley, A. (2000b). Response timing and coordination in fencing: A comparison of elite and novice fencers. Journal of Science and Medicine in Sports, 3, 460-475.

Yiou, E., \& Do, M. C. (2000). In fencing, does intensive practice equally improve the speed performance of the touché when it is performed alone and in combination with the lunge? International Journal of Sport Medicine, 21, 122-126. 